\title{
Biomechanics of Overhand Throwing Motion: - Past, Present, and Future Research Trend -
}

\author{
Shinji Sakurai*(Chukyo University)
}

Key Words: 3-D ANALYSIS, KINEMATICS, KINETICS, LINK SEGMENT MODEL

HOW was throwing motion studied in the Past?

Only humans can throw. In a more accurate sense, man is the only creature who can throw with an overhand motion. Overhand throwing motion has been extensively studied in Korea, Japan, and the USA using a variety of methods, with many research papers published especially on baseball pitching.

However, most of the research works were conducted in two dimensions, due to the restriction of the image analysis procedures available, although the three-dimensional (3-D) nature of the overhand throwing motion was well documented and recognized. Feltner and Dapena (1986) analyzed the motion of the throwing arm of baseball pitchers using DLT procedures and 3-D cinematography. Their study included both kinematic and kinetic analyses. Their study was, as a turning point, followed by many 3-D studies on the motion of the throwing arm.

\footnotetext{
투 고 일 : 2004년 1월 25일 접수

심 사 일 : 2004 년 3 월 8 일

심사완료일 : 2004년 4월 2일

* Corresponding Author, Professor, School of Health and Sports Sciences, Chukyo University, Japan 연락치 : ssakurai@sass.chukyo-u.ac.jp
} 
WHAT is a "GOOD" throwing motion?

It is well known that there is a remarkable difference in throwing ability between males and females. The gender difference in the throwing ability is much more obvious compared to other motions, such as running and jumping. The tendency that males are superior to females in throwing ability appears at a pre-school age and increases with age until adult status. We used 3-D cinematography to compare the joint angle kinematics of the throwing limb in the period up to the ball release for male and female university students throwing a softball for a distance (average distance for male: $47.0 \mathrm{~m}$, female: $22.6 \mathrm{~m}$ ). This remarkable gender difference is considered to be primarily caused by skill differences.

The throwing arm has seven degrees of freedom of joint motion apart from the fingers; three at the shoulder, one at the elbow, one at the radio-ulnar, and two for the wrist. The following seven joint angle changes corresponding to all these degrees of freedom were obtained throughout the throwing motion.

$\mathrm{J} 1$ : horizontal abduction / horizontal adduction angle at the shoulder joint,

$\mathrm{J} 2$ : abduction / adduction angle at the shoulder joint,

$\mathrm{J} 3$ : internal rotation / external rotation angle at the shoulder joint,

$\mathrm{J} 4$ : flexion / extension angle at the elbow joint,

$\mathrm{J} 5$ : pronation / supination angle at the radio-ulnar joint (forearm),

$\mathrm{J} 6$ : radial flexion / ulnar flexion angle at the wrist joint,

J7 : palmar flexion / dorsi flexion angle at the wrist joint.

Major differences in joint angle changes between male and female subjects were found in the shoulder motion (Figure 1). Male and female differed remarkably in the horizontal adduction / horizontal abduction angle of the shoulder. In the time period analyzed, the shoulder is more abducted horizontally for

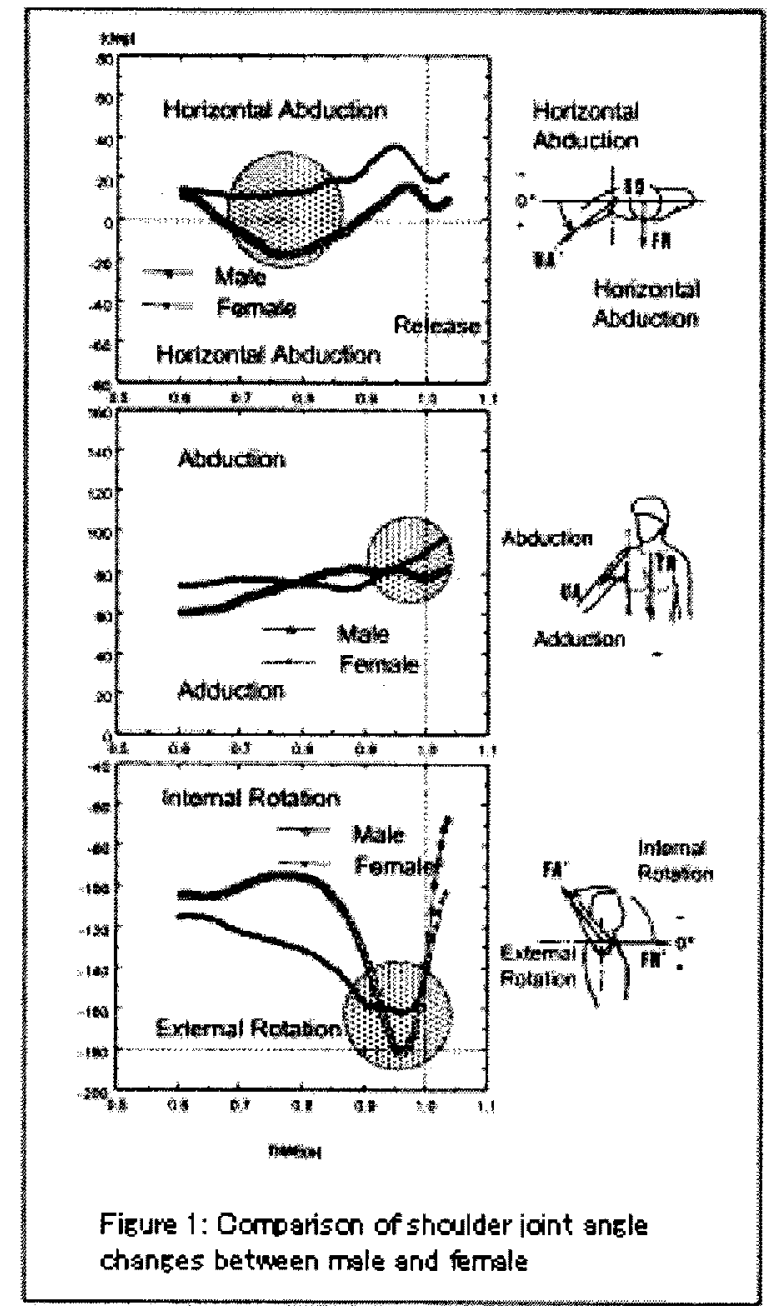


male compared to female throwers. Male subjects initially abducted the shoulder horizontally beyond a line connecting both shoulders, then adducted horizontally towards ball release. In contrast to the case of male, the mean value of the horizontal adduction angle for female subjects stayed positive indicating that the elbow was always in front of the shoulder alignment.

Though there was no statistic difference between adduction / abduction angles of shoulder between male and female subjects in the cocking phase, the shoulder was abducted and the upperarm was elevated for females in the acceleration phase, and the abduction angle for females was significantly larger than males at the ball release.

These results show the tendency of the upperarm to be raised in front of the trunk in an unskilled throwing motion. The horizontal abduction followed by horizontal adduction in the horizontal plane is a major motion in a skilled throw.

Though the shoulder joint was rotated more externally for females than males in the cocking phase, the upperarm of the male was externally rotated rapidly and the absolute value of the external rotation of the shoulder was larger than that for females just before ball release. The extreme value of the external rotation angle for the male group was -181.3 degree on average, showing that the upperarm pointed almost directlyposterior.

In four-footed animals, upperarm (and glenoid cavity) faces forward relative to the trunk, while it tends rather sideward in humans. Moreover human shoulder joint is considerably flexible because we do not need to support our body weight with the fore-limbs (arms). It might be said the skillful throwing motion of male subjects fully utilize the anatomical features of the arms and shoulders of human being.

\section{HOW is a Curveball thrown in baseball game? and HOW is it dangerous?}

Curveball pitches in baseball have been thought to increase the risk of elbow injury, particularly if the athletes begin this pitch at an early age. Some writers and researchers have claimed that overstress of the flexor and pronator muscles attached to the medial epicondyle is caused by the forearm supination required in throwing a curveball. While other researchers inferred that the curveball pitch was not as harmful as had been thought because there were no major differences in forearm muscle activity between fastball and curveball pitches. Very little quantitative data concerning the forearm and wrist action during throwing had been reported because a standard method of analysis was not established.

Joint angular kinematics of the throwing limb from the early-cocking phase to ball release were investigated for fastball and curveball baseball pitches using 3-D cinematography.

The actions were very similar for two pitches for one subject and there were no differences in the 
motions of the shoulder and elbow joints or in the temporal sequences between the two pitches. Though the forearm was more supinated at release in the curveball pitch than in the fastball pitch, both pitches were characterized by pronation of the forearm just before and after release (Figure 2). The results therefore did not support the notion that the curveball pitch is more likely than the fastball to cause elbow injuries.

\section{WHAT is necessary in the Future?}

Based on the results obtained in the studies with 3-D kinematic and kinetic analysis on rigid body link segment models, research works with computer simulation would be more frequently executed. Each body segment such as upperarm and forearm is generally modeled as a rigid body or represented by a stick, and sequence of the motion is drawn in stick

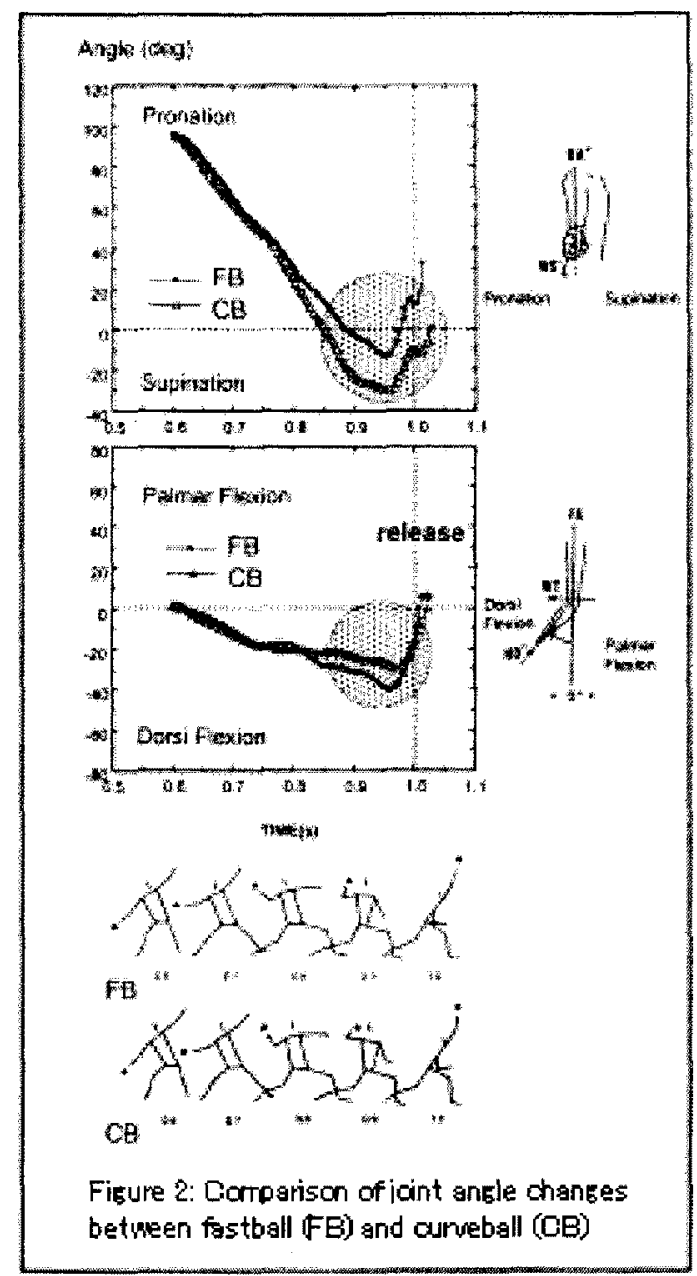
figures by joining some certain body landmarks in the motion analysis studies. For example, an upperarm is modeled as a stick joining imaginary centersof elbow and shoulder joint.

In overhand throwing the trunk and shoulder are both taking an important role as a power generator, while the hand is also playing an important role in transmittingthe momentum directly to the object. A complicated anatomical structure and fine movement skills enable us to throw skillfully. However, these regions of the human body, shoulder, trunk, and hand, are not suitable for the traditional rigid body link segment modeling.

The ratio of glenohumeral to scapular rotation is approximately $2: 1$, namely, for 180 degree of full arm elevation (abduction) the glenohumeral rotation contributes about 120 degrees and the scapular rotation around 60 degrees. Therefore, the shoulder joint angle of the throwing arm on a traditional rigid link segment model may not reflect correctly the anatomical gleno-humeral, or scapula-humerus angle.

In the future, abetter understanding of throwing motion, not only for performance enhancement but also for injury prevention, would require consideration of the movement of muscles and skeletons under 
the skin in addition to rigid link segment model analysis.

\section{CONCLUSION}

PAST:Although throwing motion is often regarded as one of the basic motions along with walking, running, and jumping, the biomechanical studies on overhand throwing are few in number compared to other motions primarily because of the restriction of analytical procedures. PRESENT: Due to the progress of the techniques of three-dimensional image analysis and kinematic and kinetic motion analysis on the rigid body segment model, overhand throwing motion has been studied widely in recent years. FUTURE: A thorough study of the role of trunk, shoulder, and hand regions are essential, and other techniques based on a musculo-skeletal modeling may also be necessary. 